\section{Principles of Modern Physics}

by Robert B. Leighton

McGraw-Hill Book Company . . \$12.50

Reviewed by. Vincent Z. Peterson

Assistant professor of physics

Many texts on atomic and nuclear physics have been written since World War II, each attempting to include the latest significant developments along with the "classical" topics of atomic physics such as natural radioactivity, $\mathrm{x}$-rays, and atomic spectroscopy. The spectacular nature of the early experimental discoveries made by Becquerel, Roentgen, J. J. Thomson, and Rutherford, and the revolutionary ideas of Einstein, Bohr, Planck, and Schroedinger make very interesting reading, as do the more recent discoveries in artificial transmutation of elements, elementary particle physics, cosmic rays, and stellar reactions. But too many books have been written as mere compendiums of assorted facts or historical accounts of celebrated experiments, without clearly stressing the interaction and significance of the discoveries. Even the better texts were destined to have a rather short "half-life," as new discoveries outmoded tables of elementary particles before they were printed, long cherished "laws" were found to have exceptions, and involved explanations of seemingly complex phenomena suddenly became clear in the light of a new theory.

The most successful textbooks covering "modern physics" on an undergraduate level have developed the important ideas through discussion of experiments, usually following th $\mathrm{e}$ historical pattern. The experimental evidence is arrayed before the theoretical interpretation is developed. An outstanding text using this approach is the Introduction to Modern Physics by Richtmyer, Kennard and T. Lauritsen (Caltecl professor of physics), last revised in 1955 (fifth edition).

The present text by Robert $B$. Leighton, associate professor of physics at Caltech, adopts a new approach -"expository and analytical, rather than historical and discursive." This is to say that the exposition of principles is at the forefront, and the chronology of events and detailed description of experiments are compressed to yield only the essential conclusions. (For example, the photoelectric effect, which receives a full chapter of experimental discussion in the usual "historical" approach, is compactly discussed in two pages of the chapter on quantum mechanics.) This approach permits a logical order of presentation of the principles, and allows sufficient space to treat some problems which are considerably more advanced than normally encountered in undergraduate texts.

The mathematical sophistication in this text is on a rather high level, particularly in subjects where significant advantages accrue to a more compact notation. One example is the use of 4-vector notation in the opening chapter on relativity. The treatment is rigorous, and the numerous problems are carefully designed to develop an intimate knowledge of the quantitative theory and to provide experience with the numerical magnitudes of atomic and nuclear quantities.

These two features - an analytical approach, and full utilization of a Caltech physics major's mathematical background - may make the b o ok rough going for a student without some prior exposure to a more elementary course in atomic and nuclear physics. For those who already have a qualitative feeling for these phenomena, however, Leighton's' approach is a significant transition to a deeper understanding of the fundamental theories of atomic and subatomic phenomena. The excellent figures, many of them original, help visualize difficult results of the theory.

The chapters on "Particles," and "Modern Physics in Nature" reflect Leighton's interests in elementary particles and cosmic rays, and are remarkably up-to-date. Mesons, hyperons and the "strangeness" theory of Gell-Mann are included; even the non-conservation of parity managed to beat the publisher's deadline!

It seems clear that the "analytical and expository approach," in the hands of a versatile experimental physicist has been successfully employed in Principles of Modern Physics.

\section{from}

\section{Deep space to Ocean floor}

\section{Vought offers this range to the young engineer}

At Chance Vought the engineer's assignments range from the depths of the ocean to the farthest reaches of space ... from hardware operating aboard the Navy's nucleararmed submarines to space research vehicles still on the boards.

Here the engineer contributes to projects such as the record-smashing Crusader jet fighter series... antisubmarine warfare studies ... missile system and space capsule development, details of which are classified.

Under the guidance of the Vought engineer, such weapons take shape. He supervises critical tests, and he introduces the weapons to the men with whom they will serve.

Engineers with many specialties share these experiences. Today, for example, Vought is at work on important projects involving:

\section{SPACECRAFT AND ASTRONAUTICS}

\section{ADVANCED PROPULSION METHODS} ELECTHONICS DESIGN AND MANUFACTURE ANTISUBMARINE WARTARE

Vought's excellent R\&D facilities help the engineer through unexplored areas. And by teaming up with other specialists against mutual challenges, the Vought engineer learns new fields while advancing in his own.

Would you like to know what men with your training are doing at Vought... what you can expect of a Vought career?

For full information, see our representative dıring his next campus visit.

\section{Or write directly to:}

C. A. Besio

Supervisor, Engineering Persornel Dept. CM-16

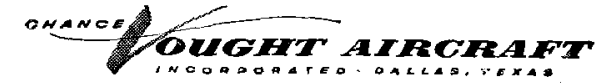

Engineering and Science 